\begin{tabular}{|c|c|c|}
\hline Beitr. Ent. & Keltern & ISSN 0005-805X \\
\hline $\mathbf{5 6}(2006) 2$ & S. $321-325$ & 15.12 .2006 \\
\hline
\end{tabular}

\title{
Eröffnung des XIX. SIEEC am 5. September 2005 in Müncheberg
}

Mit 2 Tabellen

BERNHARD KLAUSNITZER

Meine sehr verehrten Damen und Herren, liebe Gäste!

Es ist mir eine große Ehre, das XIX. Internationale Symposium für die Entomofaunistik Mitteleuropas eröffnen zu dürfen. Zum vierten Mal findet diese Tagung in Deutschland statt. Der Tagungsort - das traditionsreiche Deutsche Entomologische Institut in Müncheberg ist für Entomologen aus der ganzen Welt ein wissenschaftliches Zentrum von unersetzlichem Wert, über dessen Geschichte uns Herr Prof. Dr. DATHE einen Überblick geben wird. Es wurde erreicht, dass die wissenschaftliche (und kulturhistorische) Substanz des DEI erhalten bleibt und als Teil der deutschen zoologischen Forschung wieder institutionalisiert wurde. Das Deutsche Entomologische Institut kehrte in die Wissenschaftsgemeinschaft sogar mit einem wohlausgestatteten Neubau zurück.

Leider hat sich seit unserem XVIII. Symposium in Linz der Lebenskreis einiger unseren Tagungen besonders verbundener Entomologen vollendet, denen wir gedenken wollen. Genannt seien hier:

Vladimir Dolin, Karel Hůrka, Dalibor Povolný, Konrad Thaler.

Ich darf Sie bitten, sich zu ehrendem Gedenken zu erheben.

Besonders begrüße ich die aus 11 Ländern angereisten Gäste und danke für die zahlreichen Grüße und guten Wünsche, die unserer Tagung zugegangen sind:

Ulrike \& Horst Aspöck, Jan Carnelutti, Günther Ebert, Anna \& Hermann Levinsson, Gerd Müller-Motzfeld, Rolf Reinhardt, Gerhard Scherer, Heinrich Schmutterer, Robert Trusch, Wolfgang Zimmermann.

Seit 41 Jahren finden in Mitteleuropa Internationale Symposien zur Förderung der Entomofaunistik statt (Symposium Internationale Entomofaunisticum Europae Centralis = SIEEC). Diese Symposien hatten neben den fachlichen Zielstellungen von Anfang an auch einen besonderen humanistischen Aspekt, da sie eine Brücke zwischen Ost und West schlugen. Für viele Entomologen aus den östlichen Staaten waren sie ein wichtiger Ort, um über die Grenzen hinweg direkte wissenschaftliche Kontakte zu knüpfen und zu pflegen, die oft persönliche Akzente bekamen und manche Freundschaft begründeten. Auch wenn sich diese allgemeine Situation seit Jahren grundlegend verändert hat, bleibt die Bedeutung als Brückenschlag erhalten. 
Ein Treffen von Entomofaunisten mag dennoch heute fast als ein Anachronismus erscheinen. In einer Zeit, wo in der Zoologie das Tier im Ganzen, die eigentliche „Tierkunde“ an den Rand gedrängt erscheint und die Faunistik von den Randdisziplinen als die randlichste angesehen wird, treffen sich nun diese Leute zum 19. Mal.

Wir sind nun nicht zusammen gekommen, um das zu beklagen, uns gegenseitig zu trösten und Mut zuzusprechen - obwohl auch das dazu gehört - sondern darauf zu achten, dass das Niveau unserer Arbeit anspruchsvoll bleibt. Es muss einen Hort geben, wo sich die Kenner zusammenfinden, eine Art Tafelrunde, um die Strategien zu beraten, was man gegen die Ignoranz, das Mittelmaß und die Dummheit unternehmen kann. Damit nähren wir die Hoffnung, dass wir im Konzert der Naturwissenschaften weiter mitspielen können und zwar mit einer tragenden Melodie. Mancher Paukenschlag hat schon die Harmonie erschüttert, war aber eben nur ein temporäres Ereignis, während unser Gebiet so lange im Orchester vertreten ist, wie es überhaupt ein solches gibt. Unsere Symposien sind also nicht anachronistisch, sondern höchst aktuell. Mancher Zweifler sollte das jetzt erkennen, damit er in der Liste jener verzeichnet bleibt, denen später Weitsicht zugeschrieben werden wird.

Das erste Symposium fand im September 1964 in Opava in der damaligen ČSSR auf Initiative von ZDENEK Tesař unter der Bezeichnung „Entomologisches Symposium“ statt. Es nahmen 37 Entomologen aus 4 Ländern an dieser Tagung teil. Die Keimzelle erwies sich als lebenskräftig. Schon zwei Jahre später wurde am gleichen Ort wieder getagt, diesmal bereits mit der klar ausgewiesenen Thematik einer faunistischen und entomogeographischen Erforschung der Tschechoslowakei und Mitteleuropas. Der in Opava 1966 gefasste Beschluss über eine Weiterführung der begonnenen Tätigkeit erwies sich als tragfähig. Im Jahre 1968 wurde anlässlich des dritten Symposiums in Görlitz das Ständige Internationale Organisationskomitee gebildet, dem bei der Gründung je zwei Vertreter aus 6 Ländern angehörten. Die von dem großen Förderer unserer Symposien ZoLTAN KASZAB, Budapest, ausgearbeitete Resolution über die internationale Zusammenarbeit auf dem Gebiet der faunistischen und zoogeographischen Erforschung der Insekten Mitteleuropas war ein wichtiges Ergebnis des vierten Symposiums in Białowiecza 1969 und ist in ihren Grundzügen bis heute gültig geblieben. Seither ist die Reihe unserer Tagungen nicht abgerissen (Tabelle 1), und es besteht ein stabiler Teilnehmer- und Interessentenkreis.

Tab. 1: Liste der bisher durchgeführten Symposien (Orte und Länder in damaliger Bezeichnung).

\begin{tabular}{|l|l|r|c|}
\hline Nr. & Ort (Land) & \multicolumn{1}{c|}{ Datum } & Teilnehmerzahl \\
\hline I & Opava (ČSSR) & $22 .-24.09 .1964$ & 37 \\
\hline II & Opava (ČSSR) & $21 .-23.09 .1966$ & 74 \\
\hline III & Görlitz (DDR) & $23 .-26.04 .1968$ & 96 \\
\hline IV & Białowiecza (Polen) & $1 .-4.09 .1969$ & 75 \\
\hline V & Budapest (Ungarn) & $24 .-28.09 .1973$ & 244 \\
\hline VI & Lunz (Österreich) & $1 .-6.09 .1975$ & 85 \\
\hline VII & Leningrad (UdSSR) & $19 .-24.09 .1977$ & 266 \\
\hline
\end{tabular}




\begin{tabular}{|l|l|r|c|}
\hline Nr. & Ort (Land) & \multicolumn{1}{c|}{ Datum } & Teilnehmerzahl \\
\hline VIII & Hradec Kralove (ČSSR) & $27.08 .-2.09 .1979$ & 150 \\
\hline IX & Portoroz (Jugoslawien) & $22 .-28.06 .1981$ & 143 \\
\hline X & Budapest (Ungarn) & $15 .-20.08 .1983$ & 300 \\
\hline XI & Gotha (DDR) & $19 .-23.05 .1986$ & 250 \\
\hline XII & Kiew (UdSSR) & $24 .-30.09 .1988$ & 277 \\
\hline XIII & Gödöllö (Ungarn) & $3 .-6.09 .1991$ & 281 \\
\hline XIV & München (BRD) & $4 .-9.09 .1994$ & 205 \\
\hline XV & Iaşi (Rumänien) & $22 .-27.09 .1996$ & 123 \\
\hline XVI & Basel (Schweiz) & $14 .-18.03 .1999$ & 470 \\
\hline XVII & Radenci (Slowenien) & $20 .-26.05 .2001$ & 70 \\
\hline XVIII & Linz (Österreich) & $22 .-26.09 .2003$ & 40 \\
\hline XIX & Müncheberg (Deutschland) & $6 .-9.09 .2005$ & 60 \\
\hline
\end{tabular}

Die Zahl und das Spektrum der mitarbeitenden Länder haben sich mit der Zeit verändert und tragen der neuen Situation in Europa zunehmend Rechnung. An der Arbeit nehmen gegenwärtig folgende Länder teil: Kroatien, Niederlande, Österreich, Polen, Rumänien, Russland, Schweiz, Slowakei, Slowenien, Tschechien, Ukraine, Ungarn, Weißrussland und Deutschland.

Auf Vorschlag der ungarischen Kollegen wurde eine „Ehrenmedaille für herausragende Leistungen auf dem Gebiet der Entomofaunistik“ (In Scientia Entomofaunistica Excellenti) gestiftet und erstmals beim fünften Symposium 1973 in Budapest verliehen (Tabelle 2).

Tab. 2: Träger des Ehrenpreises für hervorragende Leistungen in der Entomofaunistik Mitteleuropas (Orte und Länder in damaliger Bezeichnung).

\begin{tabular}{|c|c|c|}
\hline Jahr & Ort & Preisträger \\
\hline 1973 & Budapest & E. LiNDNER (BRD), Z. TESAř (ČSSR) \\
\hline 1975 & Lunz & M. S. GHILARov (UdSSR), A. Horion (BRD) \\
\hline 1977 & Leningrad & M. BeIER (Österreich), Z. KASZAB (Ungarn) \\
\hline 1979 & Hradec Kralove & S. ENDRÖDI (Ungarn), J. KLIMESCH (Österreich) \\
\hline 1981 & Portoroz & W. Forster (BRD), E. Urbahn (DDR) \\
\hline 1983 & Budapest & H. Kutter (Schweiz), H. Szelegiewicz (Polen) \\
\hline 1986 & Gotha & G. A. LoHSE (BRD), R. MiKšIć (Jugoslawien) \\
\hline 1988 & Kiew & K. Harz (BRD), A. Soós (Ungarn) \\
\hline
\end{tabular}




\begin{tabular}{|c|c|c|}
\hline Jahr & Ort & Preisträger \\
\hline 1991 & Gödöllö & H. Aspöск (Österreich), I. Lopatin (UdSSR) \\
\hline 1994 & München & K. Burmann (Österreich), A. Pfeffer (Tschechien) \\
\hline 1996 & Iaşi & K. HŮRKA (Tschechien), W. MARGGI (Schweiz) \\
\hline 1999 & Basel & L. Moczar (Ungarn), B. KLAUSNitzer (BRD) \\
\hline 2001 & Radenci & Frau Heidi Günthart (Schweiz), D. PovolnÝ (Tschechien) \\
\hline 2003 & $\operatorname{Linz}$ & $\begin{array}{l}\text { B. KIs (Rumänien), Frau SARA Nógradi \& A. Uherkovich } \\
\text { (Ungarn) }\end{array}$ \\
\hline 2005 & Müncheberg & J. Carnelutti (Slowenien), K. Thaler (Österreich) \\
\hline
\end{tabular}

Zur Information und Koordinierung der Forschungsarbeiten werden im Abstand von 2-3 Jahren Symposien abgehalten. In Vorträgen werden laufende und abgeschlossene Arbeiten der teilnehmenden Länder geschildert und diskutiert. Es werden außerdem Vorschläge und Pläne für die zukünftige internationale Zusammenarbeit vorgelegt, Bedarf für Forschungsarbeiten, auch seitens der Praxis besprochen.

Hauptthemen der Arbeit der SIEEC sind:

- Förderung der Faunistik als Grundlage und Teilgebiet der Biogeographie

- Methoden der Faunistik unter besonderer Berücksichtigung der durch die Informatik gegebenen Entwicklung, insbesondere der Optimierung von Datenbanken und deren Verknüpfung

- Probleme der faunistischen Kartierung

- Dokumentation der Insektenfauna Mitteleuropas

- Erarbeitung und Herausgabe von Faunenwerken

- Förderung der taxonomischen Grundlagenforschung

- Erstellung von Spezialistenlisten der beteiligten Länder

- Förderung der regionalen Forschung (besonders auch grenzübergreifende Projekte)

Vorträge und Poster des XIX. SIEEC in Müncheberg beleuchten ganz unterschiedliche Themenbereiche, die aber alle zum Profil unserer Symposien gehören. Die behandelten Insektengruppen sind so bunt zusammengesetzt wie die Insektenwelt selbst. Natürlich gibt es - wie immer - mehrere Vorträge zur großen und gern bearbeiteten Gruppe der Coleoptera, wobei wir auf die Ausführungen zum Paläarktis-Katalog - einem Jahrhundertwerk - besonders gespannt sind. Es fehlen aber auch nicht Vorträge zu den Trichoptera, Auchenorrhyncha, Collembola, Odonata, Aphidina und Diptera. Bei den behandelten geographischen Gebieten gibt es - veranlasst durch den Tagungsort einen kleinen Schwerpunkt im Nordosten Deutschlands. Hervorzuheben sind auch die Vorträge zur Lage der Faunistik in Mitteleuropa und zu dem äußerst kontrovers diskutierten Thema des PhyloCode und DNA bar coding. Natürlich sind wir auf die Filme von Herrn Wyss sehr gespannt, diese Darbietungen werden niemanden enttäuschen.

Nun wünsche ich uns allen eine gedeihliche Zeit mit vielen interessanten Vorträgen und Postern, mit vielen Gesprächen; mögen alte Bekanntschaften und Freundschaften sich erneuern, und mögen neue Beziehungen in reicher Zahl entstehen, und möge vor allem 
der familiäre Geist sich einstellen, der unseren Symposien bisher immer eine besondere Atmosphäre verliehen hat.

Es ist beabsichtigt, die Vorträge in einem Heft der bestens renommierten, vom Deutschen Entomologischen Institut herausgegebenen Zeitschrift „Beiträge zur Entomologie“ abzudrucken. Alle Autoren von Vorträgen und einschlägigen Postern werden gebeten, entsprechend der Terminstellung und den Autorenhinweisen ihre Manuskripte rechtzeitig einzureichen.

Schon jetzt kommt ein herzlicher Dank, der zuallererst an die Veranstalter gerichtet wird. Ein ganz besonderer Dank gebührt dem Leiter des Deutschen Entomologischen Instituts Herrn Prof. Dr. Holger H. Dathe und seinen Mitarbeiterinnen und Mitarbeitern, die den Hauptanteil an der Vorbereitung und am Gelingen unseres Symposiums haben. Der Dank schließt auch alle Teilnehmer ein, die oft von weither gekommen sind und alle Referenten und Diskussionsredner, die uns mit ihren Ausführungen bereichern werden; außerdem alle, die an den Vorbereitungen für die Exkursion in den Naturpark Märkische Schweiz Anteil haben, besonders der Naturparkleiter Dr. Meinhard Ott sowie für den Gesellschaftsabend in der Stadtpfarrkirche Müncheberg, vor allem Frau IngRId PANSE, die Redakteurin Frau Anett Zimmermann und Herr Bürgermeister Klaus Zehm. Beides erwarten wir ebenfalls schon mit Spannung.

Anschrift des Verfassers:

Prof. Dr. Bernhard Klausnitzer

Lannerstraße 5

D - 01219 Dresden 